\title{
Die Abteilung Informationssysteme der Universität Oldenburg
}

\author{
H.-Jürgen Appelrath · Marco Grawunder
}

Online publiziert: 17. September 2014

(C) Springer-Verlag Berlin Heidelberg 2014

\section{Einleitung}

Die Abteilung Informationssysteme (IS) gehört zum Department für Informatik der Fakultät für Informatik, Wirtschafts- und Rechtswissenschaften an der Carl-vonOssietzky-Universität Oldenburg. IS ist mit der Gründung im Jahr 1987 aufgrund der Berufung von Prof. H.-Jürgen Appelrath eine der beiden ältesten, die bzgl. Mitarbeiterzahl größte und bzgl. ihrer Profilbildung dynamischste der aktuell insgesamt 20 Abteilungen im Department für Informatik, weil die in ihr erforschten, entwickelten und in Anwendungen evaluierten Technologien über gut 25 Jahre ungewöhnlich stark wechselten (Abb. 1).

Die sich natürlich zeitlich überlappenden Phasen der Entwicklung von IS lassen sich wie folgt charakterisieren: Von 1987 bis Anfang der 90er Jahre standen auf Basis der von Lehrstuhlinhaber und Mitarbeitern von der ETH Zürich mitgebrachten Vorarbeiten die Themen Planungssysteme und Multimedia, aber dann auch neu User-InterfaceManagement-Systeme im Vordergrund. Diese Themen wurden in den 90er Jahren ergänzt und später abgelöst durch aktive und temporale Datenbanksysteme, Data Warehouses incl. Technologien zu Datenanalyse, Datenqualitätsmanagement und Datenschutz, hier zunehmend mit Anwendungen aus Medizin und Gesundheitswesen wie z. B. in bevölkerungsbezogenen Krebsregistern [1]. Ende der 90er Jahre verstärkten sich Arbeiten zu digitalen Bibliotheken, virtuellen Laboren und generell multimedialem E-Learning, während parallele

M. Grawunder $(\bowtie) \cdot$ H.-J. Appelrath

Department für Informatik, Universität Oldenburg,

Oldenburg, Deutchland

E-Mail: marco.grawunder@uni-oldenburg.de

H.-J. Appelrath

E-Mail: appelrath@offis.de

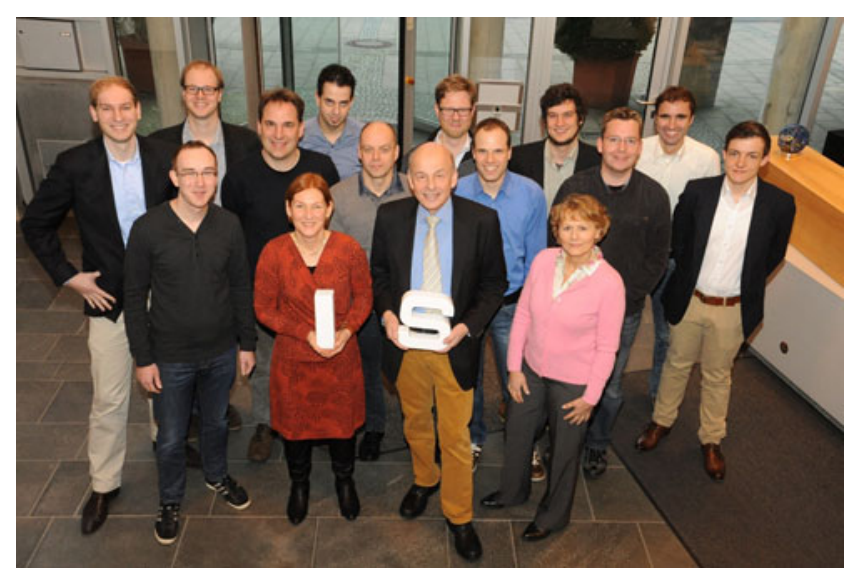

Abb. 1 Die Abteilung Informationssysteme

Aktivitäten zu betrieblichen Informationssystemen und Geschäftsprozessmanagement ausliefen.

Die Themenbereiche Geo-Informationssysteme und Mobilität in den frühen 2000er Jahren wurden schrittweise von der neu gegründeten „Nachbarabteilung“ von Susanne Boll, von 2002 bis 2006 Juniorprofessorin und seitdem auf einer ordentlichen Professur Leiterin der Abteilung „Medieninformatik und Multimediasysteme“, übernommen und intensiviert. Von 2008 bis zu ihrer Berufung an die Universität Bamberg im Frühjahr 2014 hat Daniela Nicklas auf einer Juniorprofessur „Datenbank- und Internettechnologien“ die Arbeiten der Abteilung IS verstärkt und ergänzt.

In den letzten zehn Jahren zeichnet sich die Abteilung IS durch zwei große FuE-Themen aus: Informationsmanagement in der Energiewirtschaft und Datenstrommanagementsysteme (DSMS).

Während das Thema Informationsmanagement in der Energiewirtschaft mit zunehmender Anwendungsrelevanz und durch die „Energiewende“ deutlich ausgeweitet zuneh- 
mend vom Bereich Energie im An-Institut OFFIS ${ }^{1}$ übernommen wurde, sind Technologien und Anwendungspotenziale von DSMS aktuell und absehbar im Fokus der Uni-Abteilung IS, hier vor allem der Mitarbeiter auf Landesstellen. Ergänzend gibt es derzeit und in den nächsten Jahren ein großes Drittmittelprojekt im Schnittfeld von Energiemanagement und Logistik.

Typisch für das Profil von IS war über viele Jahre die sehr enge Verzahnung mit OFFIS, vor allem in gesellschaftlich relevanten Gebieten wie Medizin, Gesundheitswesen und Energie. Seit einigen Jahren ist die Rollenverteilung zwischen dem auf rund 270 Mitarbeiterinnen und Mitarbeitern gewachsenen transferorientierten OFFIS und der grundlagenorientierter arbeitenden Uni-Abteilung IS stärker abgegrenzt, obwohl es auch immer wieder in Forschung und Lehre gemeinsame Projekte und Lehrveranstaltungen gibt. Dies zeigte sich bei der Lehre in der gesamten, über 25-jährigen Geschichte der Abteilung IS durch ein besonderes Engagement bei vielen gemeinsam getragenen Projektgruppen mit praxisrelevanten Anwendungen, aber auch bei zahlreichen studentischen Abschlussarbeiten.

\section{Anwendungsorientierte Forschungsthemen in Energiewirtschaft und Gesundheitswesen}

Die aktuellen Forschungsaktivitäten der Abteilung IS konzentrieren sich auf das Gebiet des intelligenten Datenmanagements mit den beiden Kernanwendungsgebieten Energiewirtschaft und Gesundheitswesen.

Intelligentes Datenmanagement umfasst dabei alle methodischen, konzeptionellen, technischen und organisatorischen Maßnahmen und Verfahren zur Behandlung von Daten mit dem Ziel, Informationen und schließlich neues Wissen aus den Daten zu gewinnen. Dazu gehören insbesondere Konzepte und Technologien aus den Bereichen Data Warehousing, Datenanalyse/Data Mining, Datenqualitätsmanagement sowie Datenstrommanagement.

Ein interessantes Anwendungsfeld für intelligentes Datenmanagement bieten Energiewirtschaftliche Informationssysteme. Der Energiesektor steht aufgrund der weiter steigenden Anzahl an dezentralen Energieerzeugern sowie aufgrund der Entflechtung von Erzeugung, Vertrieb und Netzbetrieb ergänzt um weitere Marktteilnehmer vor neuen Herausforderungen. Die sollen unter anderem mit neu zu schaffenden Energieinformationssystemen als Dezentrale Energiemanagementsysteme (DEMS) bewältigt werden. In DEMS findet eine Kommunikations- und Informationsintegration nicht nur der dezentralen Erzeuger, sondern auch vieler wei-

\footnotetext{
${ }^{1}$ http://www.offis.de
}

terer heterogener Akteure (wie Verbraucher, Speicher, Netze, Messstellenbetereiber etc.) und ihrer Systeme statt [3].

Intelligentes Datenmanagement ist auch eine hilfreiche Technologie bei vielen Forschungsfragen in der medizinischen Versorgung. Aufgabe der sogenannten Versorgungsforschung ist die Analyse der Gesundheitsversorgung und die Entwicklung und Evaluation neuer Versorgungskonzepte. Dadurch sollen die Versorgungsqualität verbessert und gleichzeitig die Kosten durch eine gesteigerte Effizienz des Versorgungssystems gesenkt werden.

Die stärker anwendungsunabhängigen und vorrangig technologiegetrieben FuE-Aktivitäten der Abteilung IS sind auf das im Folgenden präsentierte DSMS-Framework Odysseus konzentriert.

\section{Odysseus - Ein Framework zur Erstellung von Datenstrommanagementsystemen}

In zunehmendem Maße werden Daten nicht mehr nur auf Abruf (pull) zur Verfügung gestellt, sondern aktiv von einer Quelle als Datenstrom gesendet (push). Anwendungsgebiete sind hier das Verkehrsmanagement, die Logistik, die medizinische Überwachung, die Fertigungssteuerung (,Industrie 4.0“) oder das Energiemanagement. In allen diesen Anwendungen müssen Daten integriert, transformiert, analysiert und Ergebnisse zeitnah bereitgestellt sowie erkannte Probleme unmittelbar berichtet werden (vgl. Abb. 2).

Die Überwachung und Analyse der kontinuierlichen Datenströme findet immer noch zu oft mittels manuell erstellter Programme statt. Es wird zu viel Entwicklungszeit in Standardverarbeitungsschritte wie Einbinden von Quellen oder Zusammenführen und Aggregieren von Daten gesteckt, anstatt sich auf das eigentlich zu lösende Problem „Gewinnung von Informationen aus Daten“ zu konzentrieren. DSMS erlauben es stattdessen, diese Aufgaben mithilfe von deklarativen Anfragen/Regeln performant im Hauptspeicher zu erledigen. In Analogie zu der bereits in den 70er Jahren gewonnenen Erkenntnis, dass eine Verwaltung von Daten mit Hilfe eines Datenbankmanagementsystems einen deutlichen Vorteil gegenüber der Speicherung im Dateisystem bietet, erfolgt auch im Bereich der Verarbeitung von Datenströmen ein Umdenken. Durch die Verwendung von semantisch abstrakteren Konzepten im Rahmen einer Anfragesprache ist es deutlich schneller möglich, Anwendungen zu erstellen und einfacher zu warten. Domänenexperten werden so in die Lage versetzt, selbst Probleme zu formulieren und die Lösung anzustoßen, statt auf die Realisierung durch Entwickler zu warten. Das DSMS kümmert sich dann um Optimierungsund Skalierungsfragen.

Viele in der Forschung und auf dem Markt existierende DSMS sind als Universalwerkzeuge ausgelegt, die oft nur bestimmte Datenmodelle (i. d. R. nur das relationale) un- 
Abb. 2 Übersicht: Ereignisdatenverarbeitung

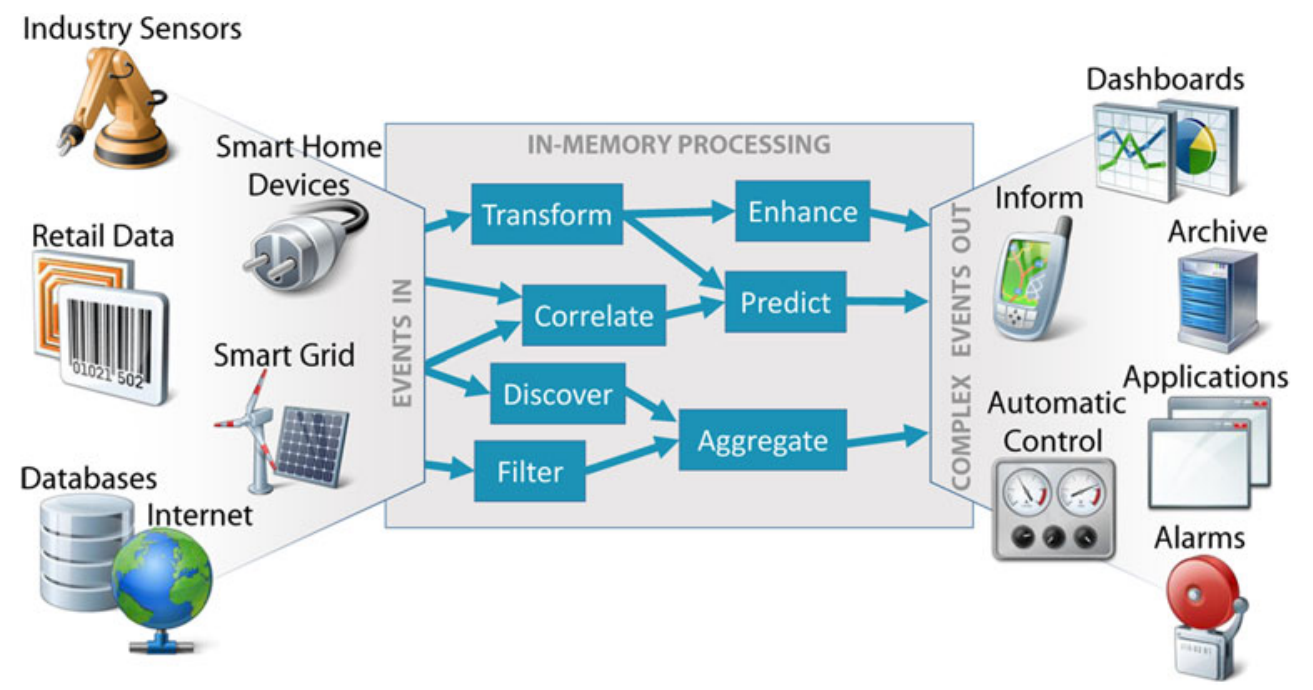

terstützen und in ihren Kernkomponenten wie bspw. dem Scheduler, der die Ausführung der Anfragen regelt, unzureichend anpassbar sind. Unsere Erfahrung hat gezeigt, dass viele Probleme im Datenstromkontext aber eine Anpassung des Systems verlangen. Dies kann zum einen das Datenmodell sein (bspw. JSON, XML oder RDF [4]), die Art der Datenverarbeitung (z. B. prioritätsbasiertes Scheduling [19] oder out-of-order, prioritätsgesteuerte Verarbeitung wichtiger Elemente [13]), aber insbesondere auch die Frage, welche Operationen auf den Daten zur Verfügung stehen und welche Quellentypen dem System bekannt sein müssen.

Aus diesem Grund entwickeln wir in IS im Projekt Odysseus seit 2007 ein gleichnamiges OSGi-basiertes Framework [2, 5, 6] für die Erstellung von Datenstrommanagementsystemen, dessen Plug-in-basierte Architektur auf besonders einfache Weise erlaubt, einzelne Komponenten anzupassen und sogar das zugrundeliegende Datenmodell auszutauschen bzw. unterschiedliche Datenmodelle gleichzeitig innerhalb einer Anfrage zu adressieren. Das wesentliche Ziel von Odysseus war und ist die Entwicklung einer Softwareplattform zur Evaluation von Verfahren der Datenstromverarbeitung.

Zur Integration von externen Quellen bietet Odysseus ein modulares und erweiterbares Adapter-Framework, in dem unterschiedliche Ebenen der Verarbeitung (Transportschicht wie TCP, File, MessageBus, Protokollschicht wie CSV, JSON, ByteBuffer) voneinander getrennt werden und durch Kombination zu passenden Wrappern werden. Weiterhin existiert ein auf Erweiterbarkeit ausgelegtes Operatorenmodell, in dem die Verarbeitung durch das Framework übernommen wird und nur nur die eigentliche Verarbeitungslogik (z. B. für einen Filter) realisiert werden muss.

Odysseus hat sich im Laufe der Jahre zu einer idealen Plattform entwickelt, um unterschiedliche Probleme der Datenstromverarbeitung zu adressieren [9]. So wurde es u. a. für die Erstellung von Fahrerassistenzsystemen [7, 8], die Über- wachung im häuslichen Kontext [10, 11] und im Bereich maritimer Sicherheit eingesetzt. Aktuelle Forschungsfragestellungen betrachten $\mathrm{u}$. a. die Verknüpfung von Recommenderfunktionalitäten, die verteilte Ausführung in einem Peer2-Peer-Netzwerk [17] sowie die Bereitstellung von Datenstromverarbeitungsmechanismen als Services [13, 14, 19].

Weitere Informationen, der Quellcode, ein Supportforum sowie herunterladbare Versionen für verschiedene Betriebssysteme finden sich unter http://odysseus.informatik.uni-oldenburg.de.

\section{Weitere Forschungsarbeiten}

Neben den Arbeiten zu Odysseus gibt es in der Abteilung IS eine breite Palette weiterer Projekte, aus denen im Folgenden eine Auswahl beschrieben werden soll.

\subsection{Process Mining: PMCube}

In der Versorgungsforschung stehen komplexe, patientenbezogene und sich oft über Jahre erstreckende, von unterschiedlichen Leistungsträgern erbrachte Prozesse der Gesundheitsversorgung im Blickpunkt. Diese müssen - i. d. R. manuell - zunächst datenschutzkonform ermittelt und beschrieben werden.

Eine Alternative zur manuellen Erhebung der Versorgungsprozesse stellt das Process Mining dar. Darunter sind Techniken zum automatischen Extrahieren, Analysieren und Manipulieren von Prozessmodellen zu verstehen. Diese sind unter anderem in der Lage, aus den während der Ausführung von Prozessen aufgezeichneten Ereignisdaten (Event Logs) automatisch entsprechende Modelle zu extrahieren, die das beobachtete Verhalten beschreiben. Da es sich bei den Event Logs jedoch um flache Tabellen handelt, die alle zu einem 
Prozess aufgezeichneten Events beinhalten, bietet Process Mining zunächst lediglich eine Gesamtsicht auf den Prozess. Für die Versorgungsforschung ist aber vor allem eine differenzierte Analyse von verschiedenen Patientengruppen (z. B. mit unterschiedlichem Alter oder Vorerkrankungen) von Interesse. Zudem ist im Vorhinein meist nicht bekannt, worin Unterschiede zwischen den einzelnen Patientengruppen liegen könnten. Daher ist eine explorative Analyse der Prozesse wünschenswert, die einem Nutzer die schrittweise Analyse und den Vergleich unterschiedlicher Patientengruppen ermöglicht.

Die Grundidee hinter dem Ansatz zum multidimensionalen Process Mining ist, dass sich die Eigenschaften der Patientengruppen, die als Attribute im Event Log stehen, als Dimensionen interpretieren lassen, die gemeinsam einen multidimensionalen Datenwürfel aufspannen. Mittels OLAPOperationen können dann beliebige Patientengruppen durch entsprechende Datenausschnitte als Datengrundlage für das Process Mining definiert werden. Somit lassen sich separate Modelle für die verschiedenen Patientengruppen erstellen, die dann vom Nutzer gegenübergestellt und miteinander verglichen werden können. Werden jedoch nicht die erwarteten Unterschiede festgestellt, kann der Nutzer die OLAP-Anfragen anpassen und entsprechend andere $\mathrm{Pa}-$ tientengruppen definieren, etwa eine Unterscheidung nach Vorerkrankungen anstatt nach Alter. Somit kann der Nutzer Schritt für Schritt die Versorgungsprozesse aus unterschiedlichen Blickwinkeln betrachten und letztendlich Auffälligkeiten darin identifizieren. Diese können dann als Ausgangspunkt für weitere Analysen dienen, indem z. B. neue Forschungshypothesen aufgestellt werden.

Ziel des Projektes PMCube [20] ist es, neue Ansätze für das multidimensionale Process Mining zu entwickeln und deren Anwendbarkeit in der Versorgungsforschung zu untersuchen. Dazu werden die Ansätze in einem Werkzeug prototypisch implementiert. Zur Evaluation soll das Werkzeug in praxisrelevanten Szenarien der Versorgungsforschung zum Einsatz gebracht und zur Beantwortung vorausgewählter Fragestellungen verwendet werden.

\subsection{Detektion unerwünschter Arzneimittelereignisse}

Ein wesentliches Ziel der Versorgungsforschung besteht in der Evaluation der Wirksamkeit von Versorgungsprozessen unter Alltagsbedingungen.

Arzneimittel sind eines der wichtigsten Instrumente der medizinischen Versorgung und stellen die Versorgungsforschung vor besondere Herausforderungen, denn jedes Medikament kann potenziell „Unerwünschte Arzneimittelereignisse“ (UAE) hervorrufen. Durch UAE ergeben sich jährliche Kosten von über 400 Mio. Euro, die das deutsche
Gesundheitssystem zu tragen hat. Darüber hinaus wird oftmals ein Zusammenhang zwischen Medikament und UAE im Versorgungsprozess übersehen, sodass die tatsächlich durch UAE entstehenden Kosten infolge von Fehldiagnosen und falschen Folgebehandlungen weitaus höher sind. Derzeit mangelt es allerdings an geeigneten Unterstützungssystemen für die UAE-Detektion. Insbesondere vor dem Hintergrund, dass Arzneimittel im Durchschnitt rund 100 (!) Nebenwirkungen haben und chronisch, unter mehreren Krankheiten leidende Patienten mehrere Medikamente einnehmen, wird der Handlungsbedarf für die Versorgungsforschung unterstrichen.

Aus informationstechnischer Perspektive ist die Tendenz zu beobachten, dass Diagnosen, Medikationen und weitere klinische Daten zunehmend digital in der „elektronischen Patientenakte“ (EPA) erfasst werden. Die Spannweite reicht dabei von rein administrativen Abrechnungsdaten bis hin zu vollständigen, lebenslangen Krankheitsverläufen, was derzeit in den meisten Ländern noch nicht realisiert ist. In einer soliden EPA verbirgt sich ein immenses Analysepotenzial, das bislang unzureichend ausgeschöpft wird. Durch eine aktive, natürlich datenschutzkonforme, weil anonymisierte Überwachung, Integration und übergreifende Analyse mehrerer EPA könnten Ärzte bei der Identifikation von UAE proaktiv unterstützt werden. Während zwar bereits interdisziplinär Ansätze zur UAE-Detektion auf klinischen Patientendaten erforscht wurden, haben existierende Arbeiten primär das Defizit, spezifisch auf Basis der zugrunde liegenden Daten konzipiert worden zu sein. Hierauf zielt die Forschungsfrage unter entsprechenden Anteilen der Abteilung IS ab, wie EPA zur Identifizierung von UAE genutzt werden können, um automatisiert und flexibel UAE zu detektieren und die Verdachtsfälle anschließend in geeigneter Form einer medizinischen Fachkraft zu präsentieren [15].

\subsection{Analytische Informationssysteme}

Im Bereich Business Intelligence (BI) haben sich Analytische Informationssysteme (AIS) entwickelt mit dem Ziel, Daten aus verschiedenen Datenquellen integriert analysieren zu können und Informationen zu gewinnen, die Entscheidungsträger, sogenannte Business User, in ihrem Entscheidungsfindungsprozess unterstützen. Sowohl die hohe Nutzungskomplexität, die sich aus der Flexibilität und Mächtigkeit solcher Systeme ergibt, als auch die notwendige intensive Interaktion mit dem Benutzer zur Durchführung adäquater Analysen erfordern i. d. R. entsprechendes domänenspezifisches Analysewissen sowie ein tiefergehendes konzeptionelles und technisches Verständnis.

Dieses ist bei Entscheidungsträgern oftmals nicht gegeben, wodurch eine eigenständige Informationsversorgung 
mittels AIS behindert wird. AIS sind somit als Systeme zu verstehen, in denen Analysten im Auftrag von Entscheidungsträgern Informationen zu gegebenen Fragestellungen ableiten. Problematisch ist in diesem Zusammenhang, dass Analysten auf neue Anfragen oftmals nicht zeitnah reagieren können und teils unpassende Informationen liefern, was unter Umständen wiederum auf sich häufig ändernde fachliche Anforderungen zurückzuführen ist. Somit besteht seitens der Entscheidungsträger der Wunsch, sich eigenständig, zeitlich unabhängig, in einer gewünschten Form mit passenden Informationen im Entscheidungsfindungsprozess versorgen zu können, ohne auf die Unterstützung von Analysten angewiesen zu sein.

Versierte Entscheidungsträger behelfen sich oft mit eigenständigen „Insel-Lösungen“ bspw. auf Basis von Microsoft Excel, was zu BI-Artefakten führt, die nicht einheitlich in der Organisation durch IT verwaltet werden können. Ansätze aus dem Bereich des Self-Service BI (SSBI) versuchen mit einem Paradigmenwechsel das Problem zu lösen, indem Aufgaben zwischen Entscheidungsträgern, Analysten und der IT verlagert werden. Für eine Informationsselbstversorgung müssen BI-Werkzeuge um benutzerfreundliche Funktionalitäten zur Ableitung von Informationen erweitert werden, aber auch die Überwachung der BI-Werkzeuge selbst nimmt einen größeren Stellenwert ein.

Ziel des Projektes KNOBI -,,Knowledge-based Business Intelligence" [16] - ist die Entwicklung einer Methode, die ein bestehendes AIS um SSBI-Konzepte erweitert. Idealerweise sollen Entscheidungsträger auf Basis einer Analyseunterstützung des AIS dazu befähigt werden, eigenständig adäquate Analysen zu bestehenden Fragestellungen durchzuführen, ohne zwingend über domänenspezifisches, technisches und methodisches Analysewissen verfügen zu müssen. Aber auch Analysten sollen in ihrer täglichen Arbeit unterstützt werden. Analyseunterstützende Funktionalitäten können dabei von weiterführenden Kontextinformationen, über eine Suchfunktion von relevanten Entitäten und eine Navigationsunterstützung in Analysepfaden bis hin zu einer Vorschlagsgenerierung für weiterführende Analyseschritte reichen. Konzeptionell und technisch soll dies mit Hilfe einer semantischen Metadatenschicht sowie ausnutzenden Diensten zur Umsetzung der SSBI-Konzepte als Erweiterung eines AIS realisiert werden. Evaluiert wird KNOBI anhand des am OFFIS entwickelten AIS MUSTANG in den Domänen „Krankenhausmarktanalyse“ und „Kommunale Datenanalyse“.

\subsection{Batterie-Elektrische Schwerlastfahrzeuge im Intelligenten Containerterminalbetrieb (BESIC)}

In BESIC, einem „Leuchtturmprojekt“ des Bundesministeriums für Wirtschaft und Energie, [12, 18] wird versucht, einen Nachweis dafür zu erbringen, dass die Elektromobi- lität mit einem Batteriewechselkonzept bei geschlossenen Transport- und Logistiksystemen betriebswirtschaftlich lohnenswert ist. Es soll aufgezeigt werden, dass sich in einem Anwendungskontext wie dem Containerterminal Altenwerder in Hamburg ein Wettbewerbsvorteil durch die Einführung von Elektrofahrzeugen ergeben kann. Ausschlaggebend dafür werden aller Voraussicht nach die Betriebskosteneinsparungen sein, die sich aus der Umstellung von dieselbetriebenen auf batterie-betriebene Transportfahrzeuge mit intelligenten Ladestrategien für die Wechselbatterien erreichen lassen.

Das Logistikgeschehen im Hafen birgt bei gesteuertem Laden gewisse zeitliche Lastverschiebungspotenziale, die seitens des Terminalbetreibers im Austausch mit Stromlieferanten vermarktet werden können. Auf diese Weise lassen sich unter anderem Vergünstigungen im Strombezug erzielen, die einem Batteriewechselkonzept mit Berücksichtigung einer Restwertminderung bei den Fahrzeugbatterien zum Durchbruch verhelfen können. Das Batteriewechselkonzept sorgt im wahrsten Sinne für eine Speicherfähigkeit im Industrieprozess bei der Frage der Bereitstellung von Prozessenergie, also in diesem Falle Traktionsenergie (,Strom zum Fahren").

Das BESIC-Vorhaben setzt exemplarisch beim geschlossenen Logistiksystem des Umschlags zwischen Seeweg und Containerlager im Containerterminal Altenwerder in Hamburg an, welcher mit seiner Kompaktbauweise als „State of the art" gilt, weil es weltweit die am weitesten automatisierte Systemlösung für die Containerlogistik darstellt. Die Grundlage für den hohen Automatisierungsgrad bildet unter anderem ein IKT-basiertes Steuerungssystem für führerlose Transportfahrzeuge im Containerterminal, das modular in ein gebräuchliches Terminalleitsystem (TLS) integriert werden konnte. Es wird für die Elektromobilität darauf ankommen, neben den leittechnischen Modulen zur Vergabe von Transportaufträgen und der Bestimmung von Transportwegen auch den Batterieeinsatz und die Ladevorgänge ganzheitlich zu planen und zu steuern.

In BESIC wird der Stellenwert von IKT-Systemen für die Netzintegration von Elektrofahrzeugen sowie für Beiträge zur Energiekosteneinsparung im gewerblichen Kontext betont, indem der Strombezug zum Laden der Wechselbatterien bezogen auf den Wirk- und Regelleistungsmarkt intelligent ausgestaltet wird.

Es wird ein „Smart Charging“-Ansatz verfolgt, dessen Planungsgrundlagen und Vorhersageinformationen wie zum Beispiel Schiffsankunftslisten und die Anzahlen von Containerbewegungen in Import- oder Exportrichtung aus bestehenden Datenbanken des Containerterminals gewonnen werden.

Weiterhin wird in BESIC untersucht, ob das Batteriewechselkonzept zur zentralen Bereitstellung von Traktionsenergie bei geschlossenen Transport- und Logistiksystemen 
mit offenen Fahrbereichen und chaotischer Lagerhaltung gewinnbringend anzuwenden ist. Elektrofahrzeuge im Containerterminal können eben nicht mit der Abfertigung von Transportaufträgen aussetzen und an eine Ladestation gestellt werden, denn dann würden weitere Transportfahrzeuge gebraucht.

Die Abteilung IS ist mit vier Wissenschaftlichen Mitarbeitern an BESIC beteiligt, die unter dem Dach des Energieforschungszentrum Niedersachsen (EFZN) mit der TU Clausthal und der Universität Göttingen wie auch industriellen Partnern aus den Bereichen Hafenlogistik, Elektrofahrzeuge und Energiewirtschaft zusammenarbeiten.

\section{Literatur}

1. Appelrath HJ, Michaelis J, Schmidtmann I, Thoben W (1996) Empfehlungen an die Bundesländer zur technischen Umsetzung der Verfahrensweisen gemäß Gesetz über Krebsregister (KRG). In: Informatik, Biometrie und Epidemiologie in Medizin und Biologie, S. $101-110$

2. Appelrath HJ, Geesen D, Grawunder M, Michelsen T, Nicklas D (2012a) Odysseus: A Highly Customizable Framework for Creating Efficient Event Stream Managemestems. In: ACM DEBS 2012. ACM

3. Appelrath HJ, Kagermann H, Mayer C (2012b) Future Energy Grid - Migrationspfade ins Internet der Energie. Springer-Verlag, Heidelberg

4. Bolles A, Grawunder M, Jacobi J (2008) Streaming SPARQL - Extending SPARQL to process data streams. In: ESWC 2008. Springer Verlag

5. Bolles A, Grawunder M, Jacobi J, Nicklas D, Appelrath HJ (2009) Odysseus: Ein Framework für maßgeschneiderte Datenstrommanagementsysteme. In: Informatik 2009, S. 2000-2014. Köllen Druck+Verlag

6. Bolles A, Geesen D, Grawunder M, Jacobi J, Nicklas D, Appelrath HJ (2010) Sensordatenverarbeitung mit dem Open Source Datenstrommanagementframework Odysseus. In: Informatik 2010, S. 404-409. Gesellschaft für Informatik. Bonn, Köllen Druck+Verlag

7. Bolles A, Geesen D, Grawunder M, Jacobi J, Nicklas D, Appelrath HJ, Köster F, Marco H (2011) StreamCars - Datenstrommanagementbasierte Verarbeitung von Sensordaten im Fahrzeug. In: BTW 2011, S. 710-713
8. Bolles A, Appelrath HJ, Geesen D, Grawunder M, Hannibal M, Jacobi J, Köster F, Nicklas D (2012) StreamCars: A New Flexible Architecture for Driver Assistance Systems. In: Intelligent Vehicles Symposium (IV 2012), S. 252-257. IEEE

9. Geesen D, Grawunder M (2012) Odysseus as Platform to Solve Grand Challenges. In: ACM DEBS 2012. ACM

10. Geesen D, Brell M, Grawunder M, Nicklas D, Appelrath HJ (2012) Data Stream Management in the AAL - Universal and Flexible Preprocessing of Continuous Sensor Data. In: Ambient Assisted Living. Springer Verlag, Heidelberg, S. 213-228.

11. Geesen D, Appelrath HJ, Grawunder M, Nicklas D (2013) Challenges for Personal Data Stream Management in Smart Buildings. In: Creating Personal, Social, and Urban Awareness through Pervasive Computing. IGI Global, Hershey, S. 201-219.

12. Ihle N, Runge S, Grundmeier N, Meyer-Barlag C, Appelrath HJ (2014) Development of an IT-architecture for the usage of flexible loads in a container terminal. In: Tagungsband der EnviroInfo 2014 ICT for energy efficiency

13. Jacobi J, Bolles A, Grawunder M, Nicklas D, Appelrath HJ (2010) A physical operator algebra for prioritized elements in data streams. Comput Sci - Res Dev 25: 235-246

14. Jacobi J, Bolles A, Grawunder M, Nicklas D, Appelrath HJ (2009) Priorisierte Verarbeitung von Datenstromelementen. In: BTW 2009. Gesellschaft für Informatik, Bonn, S. 387-406

15. Krahn T, Müller F, Appelrath HJ (2013) Detektion unerwünschter Arzneimittelereignisse - Transparenzsteigerung in der Versorgungsforschung. Tech. rep.

16. Mertens M, Krahn T, Appelrath HJ (2013) Utilizing Structured Information from Multiple External Sources in the Context of the Multidimensional Data Model. In: BIS 2013. Springer, Heidelberg, S. $88-99$

17. Michelsen T (2014) Data Stream Processing in Dynamic and Decentralized Peer-to-peer Networks. In: Proceedings of the 2014 SIGMOD PhD symposium, Snowbird, Utah, USA, S. 1-5. Universität Oldenburg

18. Runge S, Appelrath HJ, Busse S, Kolbe LM, Beck HP, Benger R (2013) Containerterminalbetriebe als Wegbereiter für Elektromobilität Herausforderungen für die IKT beim Management batterieelektrischer Schwerlastverkehre. In: Tagungsband der 5. BUISTage IT-gestütztes Ressourcen- und Energiemanagement. Springer Vieweg, Oldenburg, S. 271-282

19. Vogelgesang T, Geesen D, Grawunder M, Nicklas D, Appelrath HJ (2012) Scheduling von Datenströmen auf der Basis von Service Level Agreements. Datenbank-Spektrum 12(1): 23-32

20. Vogelgesang T, Appelrath HJ (2013) Multidimensional process mining: a flexible analysis approach for health services research. In: EDBT '13 Workshops. ACM, New York 